\title{
Preliminary Modeling, Control, and Trajectory Design for Miniature Autonomous Tailsitters
}

\author{
Nathan B. Knoebel \\ Brigham Young University - Provo, nathan.knoebel@gmail.com \\ Stephen R. Osborne \\ Brigham Young University - Provo, srosborne@gmail.com \\ Deryl Snyder \\ Brigham Young University - Provo \\ Timothy W. McLain \\ Brigham Young University - Provo, mclain@byu.edu \\ Rgllpryathis. Bndadditional works at: https://scholarsarchive.byu.edu/facpub

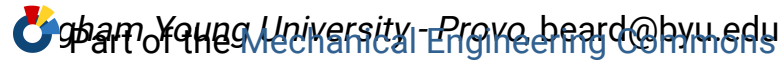

\section{Briaipal Pagelicatignicitationors}

Nathan Knoebel, Stephen Osborne, Deryl Snyder, Timothy Mclain, Randal Beard, and Andrew

Eldredge. "Preliminary Modeling, Control, and Trajectory Design for Miniature Autonomous

Tailsitters", AIAA Guidance, Navigation, and Control Conference and Exhibit, Guidance, Navigation, and Control and Co-located Conferences. http://dx.doi.org/10.2514/6.2006-6713

\section{BYU ScholarsArchive Citation}

Knoebel, Nathan B.; Osborne, Stephen R.; Snyder, Deryl; McLain, Timothy W.; Beard, Randal W.; and Eldredge, Andrew Mark, "Preliminary Modeling, Control, and Trajectory Design for Miniature Autonomous Tailsitters" (2006). Faculty Publications. 1515. https://scholarsarchive.byu.edu/facpub/1515

This Peer-Reviewed Article is brought to you for free and open access by BYU ScholarsArchive. It has been accepted for inclusion in Faculty Publications by an authorized administrator of BYU ScholarsArchive. For more information, please contact ellen_amatangelo@byu.edu. 
Authors

Nathan B. Knoebel, Stephen R. Osborne, Deryl Snyder, Timothy W. McLain, Randal W. Beard, and Andrew Mark Eldredge 


\title{
Preliminary Modeling, Control, and Trajectory Design for Miniature Autonomous Tailsitters
}

\author{
Nathan B. Knoebel Stephen R. Osborne Deryl O. Snyder* \\ Timothy W. McLain Randal W. Beard Andrew M. Eldredge \\ Brigham Young University, Provo, UT 84602
}

\begin{abstract}
A tailsitter UAV has unique advantages over typical fixed wing aircraft or hovercraft. This paper highlights topics of interest in our preliminary research in developing a tailsitter UAV. An aerodynamic model and quaternion-based attitude and position control scheme is presented for controlling a tailsitter through hover maneuvers, with simulation results. Desired trajectories are also developed through feedback linearization of the dynamic equations, intended for quaternion-based attitude control. Finally, a hardware platform is proposed.
\end{abstract}

\section{Nomenclature}

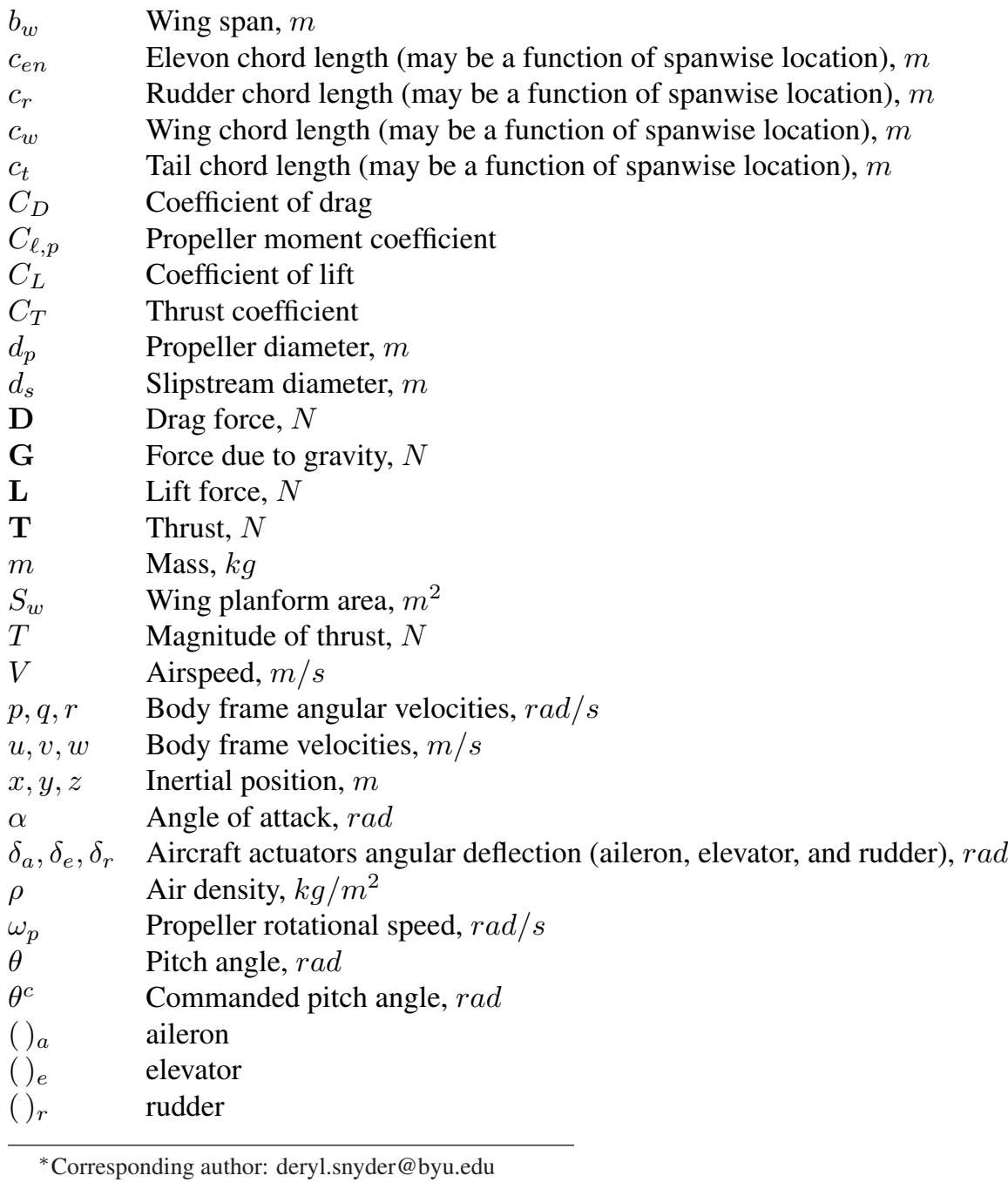




$\begin{array}{ll}()_{e n} & \text { elevon } \\ ()_{p} & \text { propeller } \\ ()_{x} & \text { body } x \text { direction } \\ ()_{y} & \text { body } y \text { direction } \\ ()_{z} & \text { body } z \text { direction }\end{array}$

\section{Introduction}

Control design for unmanned air vehicles (UAVs) is currently an active research design problem. UAVs are typically classified as fixed-wing or hovercraft. The advantages of using a fixed-wing UAV are that they are generally less expensive and more energy efficient, which implies longer flight time. However, fixed-wing UAVs cannot hover for surveillance purposes and require large areas for takeoff and landing. The advantages of hovercraft include the ability to hover and takeoff and land in confined environments, but they are relatively slow in cruise compared to fixed-wing aircraft.

A relatively unexplored aircraft design is the tailsitter, like the Convair XFY-1 shown in Figure 1. Tailsitters have the advantages of both fixed-wing airframes and hovercraft. In cruise mode they have the efficiency of a fixed-wing aircraft. In hover mode, they have the surveillance and vertical takeoff/land capabilities of a hovercraft. The main disadvantage of the tailsitter is that it is extremely difficult and dangerous for a pilot to fly, since landing requires an over-the-shoulder back-up maneuver. However, for unmanned vehicles, this disadvantage largely disappears.

Tailsitters and VTOL aircraft have received considerable attention in recent research. Wernicke $^{1}$ surveys a variety of tailsitter designs and shows the efficiency of the tailsitter concept. Ailon ${ }^{2}$ demonstrates state-to-state motion control and stable trajectory tracking with a VTOL UAV. Martin, et al. ${ }^{3}$ devise a state tracking system for a VTOL aircraft utilizing the concept of differential flatness. Much previous work has been done on the design,

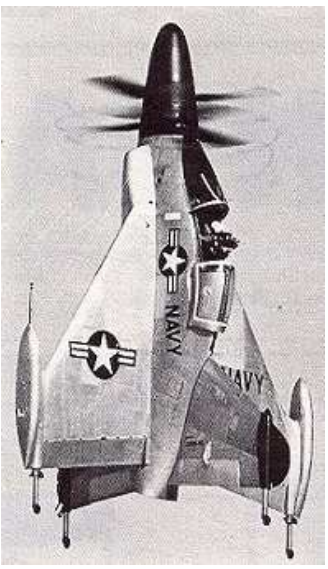

Figure 1. The experimental Convair XFY-1 "Pogo" tailsitter, first flown in 1954. control, and hardware implementation of a tailsitter UAV by Stone and Clarke. ${ }^{4-7}$ Finally, Taylor et al. ${ }^{8}$ have published a preliminary design process and lessons learned for a military autonomous VTOL system.

This paper describes the Brigham Young University Multi AGent Intelligent Coordination and Control (MAGICC) Laboratory's work on an autonomous tailsitter autopilot. First, section II discusses the simplified aerodynamic model of the tailsitter in hover mode. In section III a quaternion based attitude controller and hover position controller is discussed. Next, in section IV thrust and pitch trajectories are created from desired longitudinal motion. Finally, in section $\mathrm{V}$ a hardware platform is proposed for obtaining actual flight test results.

\section{Aerodynamic Model}

For the preliminary development of the tailsitter hover-mode controller, a simplified aerodynamic model for hover forces and moments was first derived. This model, briefly discussed in the following paragraphs, assumes the following:

- $V_{\infty}$ is very small in hover mode.

- Aerodynamic lift/drag forces due to the body are negligible.

- Aerodynamic control forces are generated only by deflections of control surfaces located within the propwash region.

- Flow in the propwash region remains parallel to the body $x$-axis.

- The only significant aerodynamic forces/moments are propeller thrust, propeller torque, lifting surface normal force (which may induce rolling moments), and lifting surface pitching moment. 

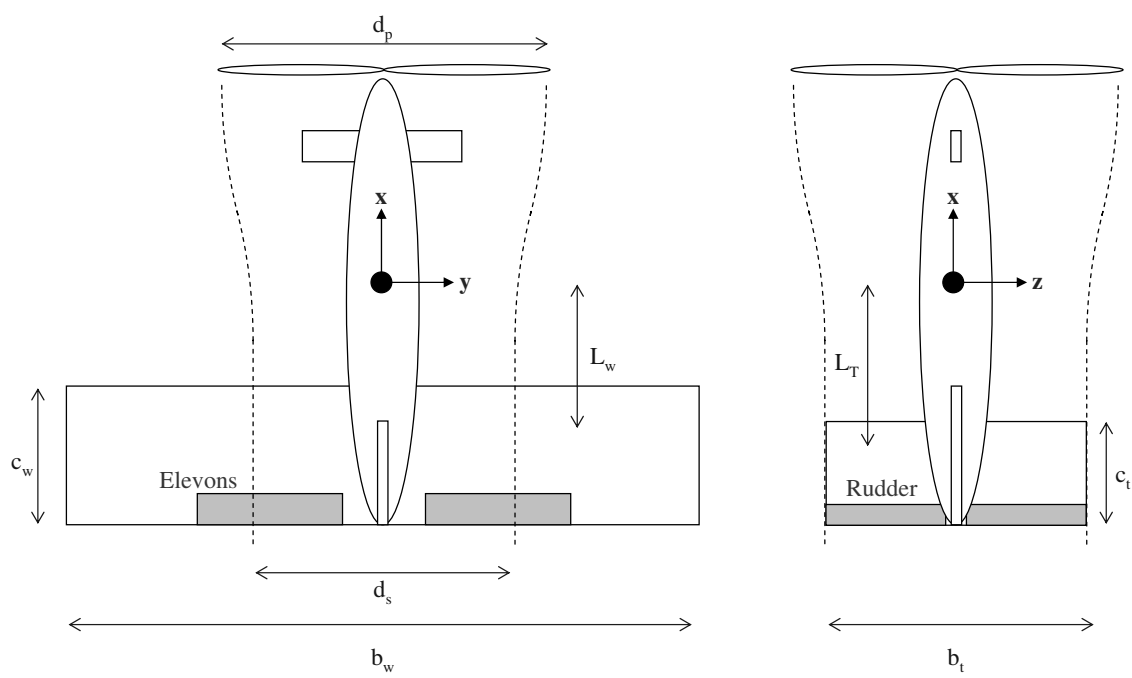

Figure 2. Sketch of tailsitter geometry and nomenclature.

Forces in the body reference frame are functions of propeller rotational speed and the angular deflection of the control surfaces:

$$
\begin{aligned}
& F_{x}=T_{p}\left(\omega_{p}\right) \\
& F_{y}=-F_{r}\left(\omega_{p}, \delta_{r}\right) \\
& F_{z}=-F_{e}\left(\omega_{p}, \delta_{e}\right)
\end{aligned}
$$

Moments, also expressed in the body reference frame, are functions of propeller rotational speed and the angular deflection of the control surfaces:

$$
\begin{aligned}
& M_{x}=M_{p}\left(\omega_{p}\right)+M_{a}\left(\omega_{p}, \delta_{a}\right) \\
& M_{y}=-F_{e}\left(\omega_{p}, \delta_{e}\right) L_{e n}+M_{e}\left(\omega_{p}, \delta_{e}\right) \\
& M_{z}=F_{r}\left(\omega_{p}, \delta_{r}\right) L_{r}+M_{r}\left(\omega_{p}, \delta_{r}\right)
\end{aligned}
$$

No aerodynamic damping is included, therefore small rotation rates are also assumed.

\section{A. Propeller}

Because we assume $V_{\infty}$ to be very small in hover mode, propeller thrust and torque coefficients are taken at their static values. The thrust produced by a propeller is found from the non-dimensional thrust coefficient:

$$
T_{p}=C_{T} \rho\left(\frac{\omega_{p}}{2 \pi}\right)^{2} d_{p}^{4}
$$

The static thrust coefficient is a function of the propeller geometry, most significantly the pitch to diameter ratio. Typical values of $C_{T}$ are on the order of 0.10 .

The moment about the body $x$-axis produced by a spinning propeller is found from the non-dimensional torque coefficient

$$
M_{p}=C_{\ell, p} \rho\left(\frac{\omega_{p}}{2 \pi}\right)^{2} d_{p}^{5}
$$

The static torque coefficient is also a function of the propeller geometry. Typical values of $C_{\ell, p}$ are on the order of 0.006 .

The velocity inside the slipstream is computed using Goldstien's momentum theory. Again, for $V_{\infty}=0$ we find

$$
V_{s}=\sqrt{\frac{2 T_{p}}{\rho A_{p}}} .
$$




\section{B. Ailerons}

Although the actual control surfaces used to produce pitching and rolling moments will be elevons, the contributions to the aerodynamic forces can be separated into aileron and elevator contributions. The ailerons produce a moment about the $x$-axis only. Using typical sign convention, a positive aileron deflection is right wing trailing-edge down, left wing trailing-edge up, which produces a rolling moment to the left, or negative about the $x$-axis. A very simple method for estimating the rolling moment induced by the ailerons, called strip theory, multiplies the local section lift increment due to the aileron deflection by the local moment arm and integrates over the wing.

The change in lift coefficient for a $2 \mathrm{D}$ wing section as a function of aileron deflection is

$$
\left(\Delta \widetilde{C}_{L w}\right)_{\delta a}= \pm \widetilde{C}_{L w, \alpha} \widetilde{\epsilon}_{e n} \delta a
$$

where $\sim$ is used to denote $2 \mathrm{D}$ section properties. For a symmetric deflection of the ailerons, strip theory gives

$$
C_{\ell, \delta a}=-\frac{2 \widetilde{C}_{L w, \alpha}}{S_{w} b_{w}} \int_{y_{i}}^{y_{o}} y \widetilde{\epsilon}_{e n} c_{w} d y
$$

We can simplify even further by assuming that the wing chord length is constant with span, as is the aileron size. This yields

$$
C_{\ell, \delta a}=-\frac{\widetilde{C}_{L w, \alpha}}{S_{w} b_{w}} \widetilde{\epsilon}_{e n} c_{w}\left(y_{o}^{2}-y_{i}^{2}\right)
$$

Finally, assuming no rolling moment is produced at zero aileron deflection, the dimensional moment produced by an aileron deflection $\delta_{a}$ is

$$
M_{a}=\frac{1}{2} \rho V_{s}^{2}\left(\widetilde{C}_{L w, \alpha} \widetilde{\epsilon}_{e n} c_{w}\left(y_{o}^{2}-y_{i}^{2}\right)\right)
$$

where $y_{i}$ is the body $y$-coordinate of the "inner" most edge of the elevon, and $y_{o}$ is the body $y$-coordinate of the "outer" most edge of the elevon or the coordinate of the outer most edge of the slipstream. The lift slope for the 2D wing sections can be approximated as

$$
\widetilde{C}_{L w, \alpha} \approx 2 \pi
$$

and from thin airfoil theory (see for instance Ref. [9]) the elevon efficiency is

$$
\widetilde{\epsilon}_{e n} \approx 1-\frac{\sigma_{e n}-\sin \left(\sigma_{e n}\right)}{\pi}
$$

with

$$
\sigma_{e n}=\cos ^{-1}\left(2 \frac{c_{e n}}{c_{w}}-1\right)
$$

\section{Elevator}

The elevators produce a force in the body $z$-direction and a corresponding moment about the body $y$-axis. Note that a positive elevator deflection is defined to be trailing-edge downward, producing a nose-down pitching moment. Again using strip theory, we integrate the section change in lift coefficient over the spanwise extent of the control surface

$$
C_{z, \delta_{e}}=\frac{2 \widetilde{C}_{L w, \alpha}}{S_{w}} \int_{y_{i}}^{y_{o}} \widetilde{\epsilon}_{e n} c_{w} d y
$$

Assuming symmetry about the $y$-axis and that the wing chord and elevon geometry are constant along the span, this yields

or

$$
C_{z, \delta_{e}}=\frac{2 \widetilde{C}_{L w, \alpha}}{S_{w}} \widetilde{\epsilon}_{e n} c_{w}\left(y_{o}-y_{i}\right)
$$

$$
F_{e}=\rho V_{s}^{2}\left(\delta_{e} \widetilde{C}_{L w, \alpha} \widetilde{\epsilon}_{e n}\right) c_{w}\left(y_{o}-y_{i}\right)
$$

with the wing lift slope and elevon efficiency defined in Eqs. (8) and (17), respectively.

Using a very similar analysis, the pitching moment about the lifting surface quarter-chord is

$$
M_{e}=\rho V_{s}^{2} \widetilde{C}_{m, \delta e} \delta_{e} c_{w}\left(y_{o}-y_{i}\right) .
$$


where, from thin airfoil theory, we can approximate

$$
\widetilde{C}_{m, \delta e}=\frac{\sin \left(2 \sigma_{e n}\right)-2 \sin \left(\sigma_{e n}\right)}{4}
$$

with $\sigma_{e n}$ defined in Eq. (10).

\section{Rudder}

The rudder produces a force in the body $y$-direction and a corresponding moment about the body $z$-axis. Note that a positive rudder deflection is defined to be trailing-edge leftward, causing the plane to yaw to the left. Using an approach similar to that employed for the elevator, and referring to the sketch in Fig. 2, we find the force produced by the rudder is

$$
F_{r}=\frac{1}{2} \rho V_{s}^{2}\left(\delta_{r} \widetilde{C}_{L t, \alpha} \epsilon_{r}\right) c_{t} d_{s} .
$$

The section lift slope is again approximated as $C_{L t, \alpha} \approx 2 \pi$ and the rudder efficiency is

$$
\widetilde{\epsilon}_{r} \approx 1-\frac{\sigma_{r}-\sin \left(\sigma_{r}\right)}{\pi}
$$

with

$$
\sigma_{r}=\cos ^{-1}\left(2 \frac{c_{r}}{c_{t}}-1\right) .
$$

Finally, the moment produced about the lifting surface quarter-chord due to rudder deflection is

$$
M_{r}=-\frac{1}{2} \rho V_{s}^{2} \widetilde{C}_{m, \delta r} \delta_{r} d_{s} c_{t} .
$$

where

$$
\widetilde{C}_{m, \delta r}=\frac{\sin \left(2 \sigma_{r}\right)-2 \sin \left(\sigma_{r}\right)}{4}
$$

\section{Quaternion Feedback Hover Control}

The quaternion attitude control structure has been explored by numerous researchers, ${ }^{4,10-12}$ however, mainly for spacecraft. Given the applications intended for the tailsitter design, quaternion based control is attractive. In the quaternion formulation, attitude error can be represented conveniently in the aircraft body frame about axes corresponding to available control actuator inputs (aileron, elevator, rudder). Also, the quaternion attitude representation lacks singularities inherent with the Euler angle formulation. Consequently, the current preliminary tailsitter hover controller being tested, shown in Figure 3, implements such control.

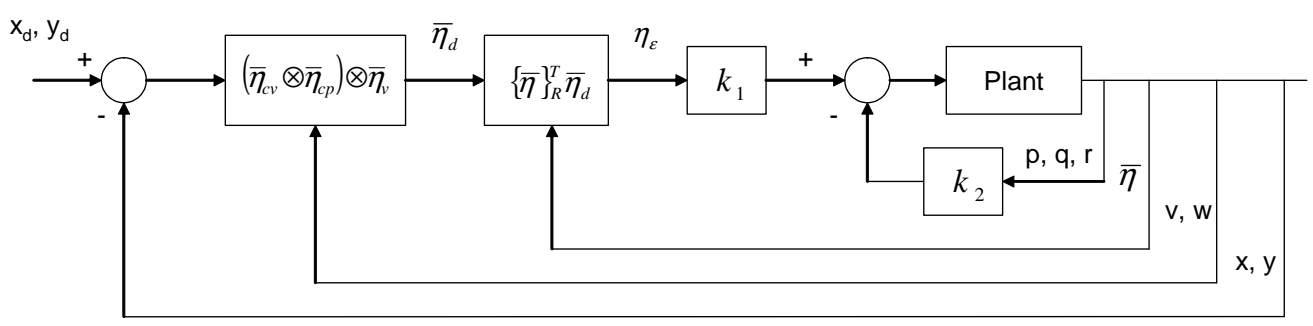

Figure 3. Hover controller block diagram

\section{A. Quaternion Attitude Control}

According to Euler's theorem, attitude can be represented as a single rotation about an axis in three-dimensional space. ${ }^{13}$ The quaternion vector used to represent the aircraft is of the form

$$
\bar{\eta}=\left(\begin{array}{c}
\eta \\
\eta_{4}
\end{array}\right)=\left(\begin{array}{llll}
\eta_{1} & \eta_{2} & \eta_{3} & \eta_{4}
\end{array}\right)^{T} .
$$


The terms $\eta$ and $\eta_{4}$ can be described in the following manner:

$$
\eta=\left(\begin{array}{l}
\eta_{1} \\
\eta_{2} \\
\eta_{3}
\end{array}\right)=\sin \left(\frac{\Theta}{2}\right) \hat{\eta} \quad \eta_{4}=\cos \left(\frac{\Theta}{2}\right)
$$

where $\hat{\eta}$ describes the axis of rotation and $\Theta$ gives the scalar angle of rotation about that axis. To preserve the unity norm, the quaternion is scaled by the angle of rotation $\Theta$ as shown. Quaternion multiplication can be defined as ${ }^{14}$

$$
\bar{\eta} \prime \prime=\bar{\eta} \prime \otimes \bar{\eta}=\left(\begin{array}{c}
\eta_{4} \eta^{\prime}+\eta_{4} \prime \eta-\eta \prime \\
\eta_{4}{ }^{\prime} \eta_{4}-\eta \prime . \eta
\end{array}\right),
$$

for quaternion rotations, where the resulting quaternion $\bar{\eta} \prime \prime$ is the result of two successive rotations represented by $\bar{\eta}$ and $\bar{\eta}$. Equation (23) can also be written as ${ }^{14}$

$$
\bar{\eta} \prime \prime=\bar{\eta} \prime \otimes \bar{\eta}=\{\bar{\eta}\}_{R} \bar{\eta} \prime
$$

where

$$
\{\bar{\eta}\}_{R}=\left(\begin{array}{cccc}
\eta_{4} & -\eta_{3} & \eta_{2} & \eta_{1} \\
\eta_{3} & \eta_{4} & -\eta_{1} & \eta_{2} \\
-\eta_{2} & \eta_{1} & \eta_{4} & \eta_{3} \\
-\eta_{1} & -\eta_{2} & -\eta_{3} & \eta_{4}
\end{array}\right)
$$

Consider the following equation,

$$
\bar{\eta}_{d}=\bar{\eta}_{\epsilon} \otimes \bar{\eta}_{a}=\left\{\bar{\eta}_{a}\right\}_{R} \bar{\eta}_{\epsilon}
$$

where $\bar{\eta}_{d}$ represents the aircraft's desired attitude, $\bar{\eta}_{a}$ represents the actual attitude, and $\bar{\eta}_{\epsilon}$ represents the error between the two expressed in the aircraft's body reference frame. Noting that $\{\bar{\eta}\}_{R}^{T}\{\bar{\eta}\}_{R}=I_{4}$, we can resolve the error quaternion as

$$
\bar{\eta}_{\epsilon}=\left\{\bar{\eta}_{a}\right\}_{R}^{T} \bar{\eta}_{d}
$$

Since the error quaternion is expressed in the aircraft body reference frame and its first three elements $\eta_{\epsilon}$, are scaled by $\sin \left(\Theta_{\epsilon} / 2\right)$, the three aircraft actuators (aileron, elevator, and rudder) can be employed directly to control $\eta_{\epsilon}$, in order to drive $\Theta_{\epsilon}$ to zero. Therefore, given a desired quaternion and existing angular rates $\omega=(p q r)^{T}$, stable attitude control for instantaneous control surface deflections and no external disturbances can be achieved by

$$
\left(\begin{array}{c}
\delta_{a} \\
\delta_{e} \\
\delta_{r}
\end{array}\right)=k_{1} \eta_{\epsilon}-k_{2} \omega
$$

where $k_{1}$ and $k_{2}$ are diagonal gain matrices.

\section{B. Hover Position Control}

For position control (assuming altitude can be controlled from throttle) a desired quaternion can be defined as

$$
\bar{\eta}_{d}=\bar{\eta}_{c} \otimes \bar{\eta}_{v}
$$

The term $\bar{\eta}_{v}$ is defined as the vertical quaternion

$$
\bar{\eta}_{v}=\left(\begin{array}{c}
0 \\
\sqrt{2} / 2 \\
0 \\
\sqrt{2} / 2
\end{array}\right)
$$

This can be understood as the aircraft nose pointing up along the negative $z$ vehicle axis and the underside pointing north along the $x$ vehicle axis. The term $\bar{\eta}_{c}$ is the primary correction quaternion that describes the rotation needed to tilt the nose of the aircraft off the vertical orientation for $x-y$ position tracking 


$$
\bar{\eta}_{c}=\bar{\eta}_{c v} \otimes \bar{\eta}_{c p}
$$

where $\eta_{c p}$ is a correction quaternion based on the position error. $\eta_{c v}$ is a correction quaternion based on body frame velocities that provides damping. The components of $\bar{\eta}_{c p}$ namely $\hat{\eta}_{c p}$ and $\Theta_{c p}$ can be obtained by

$$
\hat{\eta}_{c p}=\left(\begin{array}{l}
0 \\
0 \\
1
\end{array}\right) \times\left(\begin{array}{c}
\left(x-x_{d}\right) /\left\|e_{p}\right\| \\
\left(y-y_{d}\right) /\left\|e_{p}\right\| \\
0
\end{array}\right) \quad \Theta_{c p}=k_{3}\left\|e_{p}\right\|
$$

where $k_{3}$ is a gain and $\left\|e_{p}\right\|$ is the norm of the position error

$$
\left\|e_{p}\right\|=\sqrt{\left(x-x_{d}\right)^{2}+\left(y-y_{d}\right)^{2}} .
$$

The terms $x_{d}$ and $y_{d}$ refer to the aircraft desired position. For $\bar{\eta}_{c v}$ the terms $\hat{\eta}_{c v}$ and $\Theta_{c v}$ can be found from

$$
\hat{\eta}_{c v}=\left(\begin{array}{l}
0 \\
0 \\
1
\end{array}\right) \times\left(\begin{array}{c}
\frac{w}{\sqrt{v^{2}+w^{2}}} \\
\frac{v}{\sqrt{v^{2}+w^{2}}} \\
0
\end{array}\right) \quad \Theta_{c v}=k_{4} \sqrt{v^{2}+w^{2}}
$$

where $k_{4}$ is a gain.

\section{Hover Position Tracking Simulation}

Results found through simulation verify the control method discussed above. Figures $4 \mathrm{~b}$ and $4 \mathrm{c}$ exhibit north and east position tracking during a combined ascent, translation, and descent hover maneuver. Figure $4 \mathrm{~d}$ shows the first quaternion error element throughout the flight described above, as well as the control required to minimize that error. The simulated maneuver's altitude tracking can be seen in Figure 4a. The simulation model uses the simple hover forces and torque model described earlier and the standard translational and rotational kinematic and dynamic equations for non-linear 6-DOF rigid-body aircraft simulation. ${ }^{15}$

\section{Trajectory Generation}

Typical mission requirements for a tailsitter UAV will involve several transitions between hover and level flight. It is desirable to obtain a trajectory generation scheme that can successfully negotiate these transitions. We develop such a scheme in this section, with desired $(x, z)$ paths as inputs and thrust and pitch commands as outputs.

Restricting our analysis to the longitudinal dynamics of the vehicle, the forces acting on the tailsitter are shown in Figure 5. Accordingly, the equations of motion are given by

$$
\begin{aligned}
m\left(\begin{array}{l}
\ddot{x} \\
\ddot{z}
\end{array}\right) & =\mathbf{G}+\mathbf{L}+\mathbf{D}+\mathbf{T} \\
\dot{\theta} & =a\left(\theta^{c}-\theta\right) .
\end{aligned}
$$

In the inertial frame, the gravity vector is given by

$$
\mathbf{G}=\left(\begin{array}{c}
0 \\
-m g
\end{array}\right)
$$

We will assume that the thrust vector is directed along the $x$ axis in the body frame. Therefore, in the inertial frame we have

$$
\mathbf{T}=\left(\begin{array}{l}
T \cos \theta \\
T \sin \theta
\end{array}\right) .
$$

We will assume that $T>0$ is an input to the system. 

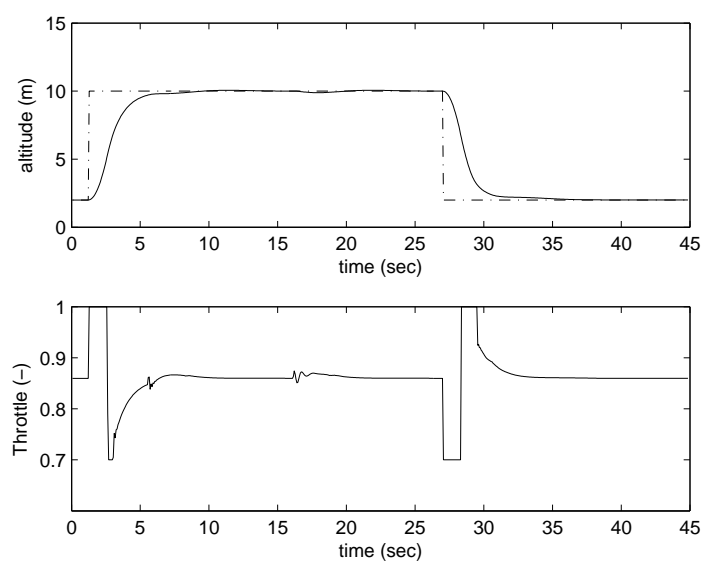

(a) Altitude
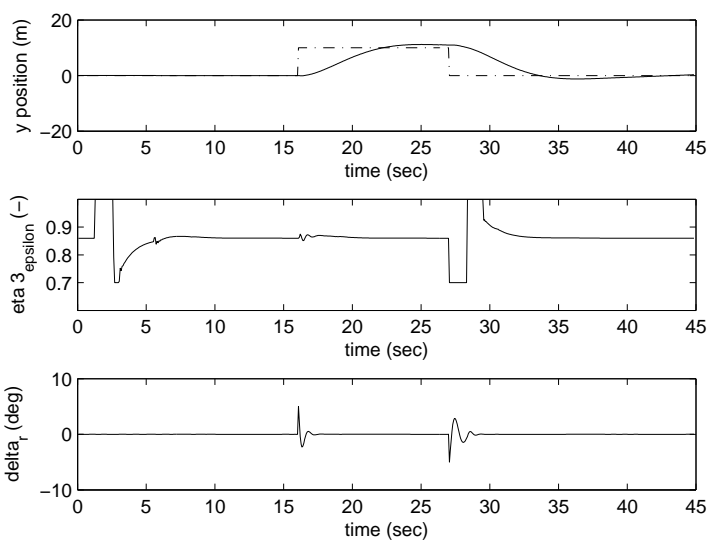

(c) East
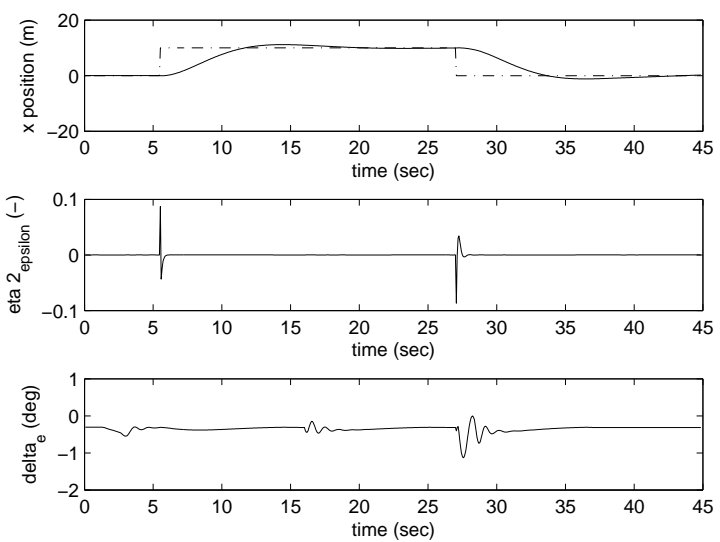

(b) North
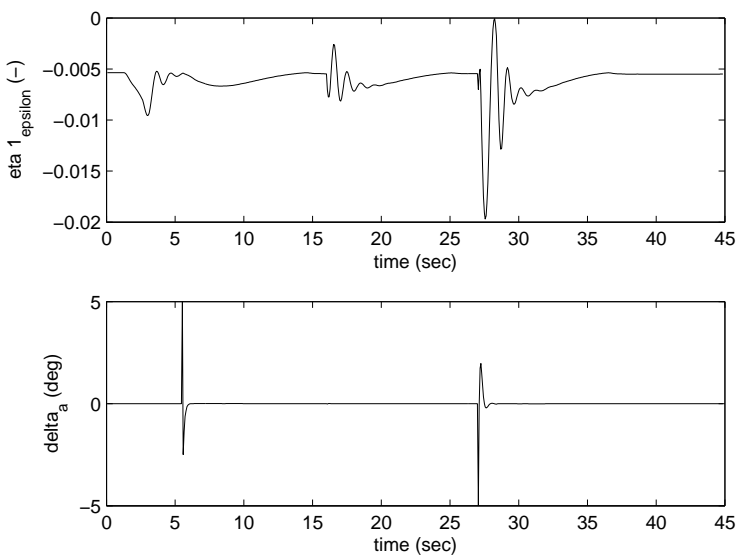

(d) $e_{\bar{\eta} 1}$

Figure 4. Simulation results of hover position controller

Similarly, the lift and drag vectors in inertial space are given by

$$
\begin{gathered}
\mathbf{L}=\frac{1}{2} \rho V^{2} S_{w}\left(\begin{array}{c}
-\sin (\theta-\alpha) \\
\cos (\theta-\alpha)
\end{array}\right) \\
\mathbf{D}=\frac{1}{2} \rho V^{2} S_{w}\left(\begin{array}{l}
-\cos (\theta-\alpha) \\
-\sin (\theta-\alpha)
\end{array}\right) .
\end{gathered}
$$

Note that the airspeed is given by

$$
V=\sqrt{\dot{x}^{2}+\dot{z}^{2}}
$$

and the angle of attack is given by

$$
\alpha=\theta-\tan ^{-1}\left(\frac{\dot{z}}{\dot{x}}\right) .
$$

Therefore $\theta-\alpha=\tan ^{-1}\left(\frac{\dot{z}}{\dot{x}}\right)$ and

$$
\cos (\theta-\alpha)=\cos \tan ^{-1}\left(\frac{\dot{z}}{\dot{x}}\right)=\frac{\dot{x}}{\sqrt{\dot{x}^{2}+\dot{z}^{2}}}=\frac{\dot{x}}{V}
$$




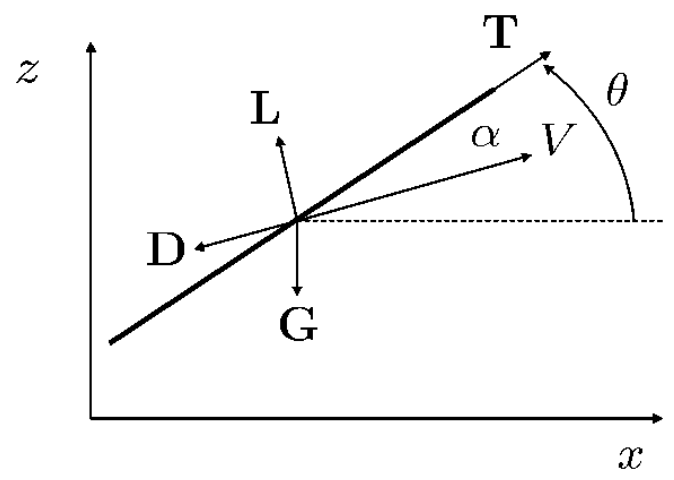

Figure 5. Description of the forces acting on the tailsitter.

$$
\sin (\theta-\alpha)=\sin \tan ^{-1}\left(\frac{\dot{z}}{\dot{x}}\right)=\frac{\dot{z}}{\sqrt{\dot{x}^{2}+\dot{z}^{2}}}=\frac{\dot{z}}{V}
$$

Then

$$
\mathbf{L}+\mathbf{D}=\frac{1}{2} \rho V^{2} S_{w}\left(\begin{array}{cc}
\frac{\dot{x}}{V} & -\frac{\dot{z}}{V} \\
\frac{\dot{z}}{V} & \frac{\dot{x}}{V}
\end{array}\right)\left(\begin{array}{c}
-C_{D} \\
C_{L}
\end{array}\right)=\frac{1}{2} \rho V S_{w}\left(\begin{array}{c}
-\dot{x} C_{D}-\dot{z} C_{L} \\
-\dot{z} C_{D}+\dot{x} C_{L}
\end{array}\right) .
$$

The equations of motion for $x$ and $z$ are rewritten as

$$
\left(\begin{array}{c}
\ddot{x} \\
\ddot{z}
\end{array}\right)=\left(\begin{array}{c}
0 \\
-g
\end{array}\right)+\frac{1}{2 m} \rho V S_{w}\left(\begin{array}{l}
-\dot{x} C_{D}-\dot{z} C_{L} \\
-\dot{z} C_{D}+\dot{x} C_{L}
\end{array}\right)+\frac{T}{m}\left(\begin{array}{c}
\cos \theta \\
\sin \theta
\end{array}\right) .
$$

\section{A. Simple model for lift and drag}

A lift and drag model is required for trajectory generation. Generic lift and drag curves for angles of attack between -90 and +90 degrees are shown in Figure 6. Although these curves do not represent any particular aircraft, they contain the major features seen in various experiments. The lift curve shows a linear change with angle of attack for angles below stall, followed by a large drop in lift after stall. For angles where significant separation exists, the lift follows the theoretical values predicted for a flat plate. The drag coefficient, on the other hand, varies smoothly from a minimum near zero angle of attack to a maximum near 90 degrees.

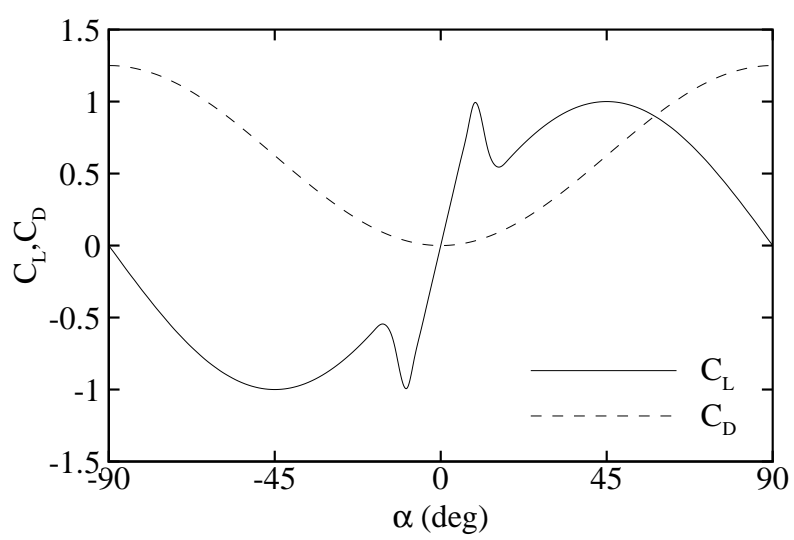

Figure 6. Simple model for lift and drag of a symmetric lifting surface for angles of attack between $\pm 90^{\circ}$ (based on the experimental results of Sheldal and Klimas ${ }^{16}$ ). 


\section{B. Feedback Linearization}

We will find inputs $T$ and $\theta^{c}$ to follow a desired trajectory $\left(x^{d}, z^{d}\right)$. Define

$$
\begin{aligned}
& \tilde{x}=x-x^{d}, \tilde{z}=z-z^{d} \\
& \dot{\tilde{x}}=\dot{x}-\dot{x}^{d}, \dot{\tilde{z}}=\dot{z}-\dot{z}^{d} \\
& \ddot{\tilde{x}}=\ddot{x}-\ddot{x}^{d}, \ddot{\tilde{z}}=\ddot{z}-\ddot{z}^{d} .
\end{aligned}
$$

Then,

$$
\left(\begin{array}{c}
\ddot{\tilde{x}} \\
\ddot{\tilde{z}}
\end{array}\right)=\left(\begin{array}{c}
0 \\
-g
\end{array}\right)+\frac{1}{2 m} \rho V S_{w}\left(\begin{array}{l}
-\dot{x} C_{D}-\dot{z} C_{L} \\
-\dot{z} C_{D}+\dot{x} C_{L}
\end{array}\right)+\frac{T}{m}\left(\begin{array}{c}
\cos \theta \\
\sin \theta
\end{array}\right)-\left(\begin{array}{c}
\ddot{x}^{d} \\
\ddot{z}^{d}
\end{array}\right) .
$$

By picking the inputs $T$ and $\theta^{c}$ such that

$$
\frac{T}{m}\left(\begin{array}{c}
\cos \theta \\
\sin \theta
\end{array}\right)=\left(\begin{array}{c}
-\frac{\rho V S_{w}}{2 m}\left(-\dot{x} C_{D}-\dot{z} C_{L}\right)+\ddot{x}^{d}-k_{d} \dot{\tilde{x}}-k_{p} \tilde{x} \\
g-\frac{\rho V S S_{w}}{2 m}\left(-\dot{z} C_{D}+\dot{x} C_{L}\right)+\ddot{z}^{d}-k_{d} \dot{\tilde{z}}-k_{p} \tilde{z}
\end{array}\right)
$$

we can cause

$$
\left(\begin{array}{c}
\ddot{\tilde{x}} \\
\ddot{\tilde{z}}
\end{array}\right)=\left(\begin{array}{l}
-k_{d} \dot{\tilde{x}}-k_{p} \tilde{x} \\
-k_{d} \dot{\tilde{z}}-k_{p} \tilde{z}
\end{array}\right)
$$

and therefore $\tilde{x} \rightarrow 0, \tilde{z} \rightarrow 0$. Let

$$
\begin{gathered}
A=\frac{\rho V S_{w}}{2 m}\left(-\dot{x} C_{D}-\dot{z} C_{L}\right)-\ddot{x}^{d}+k_{d} \dot{\tilde{x}}+k_{p} \tilde{x} \\
B=-g+\frac{\rho V S_{w}}{2 m}\left(-\dot{z} C_{D}+\dot{x} C_{L}\right)-\ddot{z}^{d}+k_{d} \dot{\tilde{z}}+k_{p} \tilde{z} .
\end{gathered}
$$

Then the desired inputs are

$$
\begin{gathered}
T=m \sqrt{A^{2}+B^{2}} \\
\theta^{c}=\tan ^{-1} \frac{B}{A} .
\end{gathered}
$$

Expressed in quaternion form, the axis of rotation for pitch is the body frame $y$ axis. Therefore the desired quaternion vector is

$$
\bar{\eta}_{d}=\left(\begin{array}{c}
0 \\
\sin \frac{\theta^{c}}{2} \\
0 \\
\cos \frac{\theta^{c}}{2}
\end{array}\right) .
$$

A Matlab Simulink simulation of the trajectory generator yields good results as shown in Figure 7. The commanded flight path is a vertical climb followed by level flight followed by a vertical descent. As seen in Figures 7d and 7f, position error remains very low throughout the simulated flight despite the nonlinear characteristics of the transitional maneuvers made from hover to level flight and from level to hover flight. It is expected that a hardware implementation would not yield such low tracking error, but the approach appears promising for such transitional maneuvers. We also see that much more thrust is required for the vertical flight regions, as expected. Figure 8 shows detail of the thrust command around the two transition regions. During the vertical-to-level transition, thrust drops suddenly as the tailsitter tips over from a vertical to a horizontal position. During the level-to-vertical transition, thrust also suddenly drops at the end of the tailsitter's gradual slide into vertical flight.

\section{Proposed Hardware Platform}

We intend to develop a working tailsitter UAV incorporating the algorithms and concepts detailed in this paper. Tailsitter RC model airplane kits are commercially available, including the Pogo ${ }^{17}$ shown in Figure 9. Lightweight construction, a powerful motor, and large control surfaces allow the Pogo to takeoff vertically, hover, or fly in level flight thus meeting our needs for a tailsitter UAV testbed. The Pogo has been equipped with the Kestrel Autopilot ${ }^{18}$ running a $29 \mathrm{MHz}$ Rabbit microcontroller with 512K Flash and 512K RAM. The sensors on the autopilot include rate gyros, accelerometers, an absolute pressure sensor for measuring altitude, a differential pressure sensor for measuring airspeed, and a GPS receiver. 


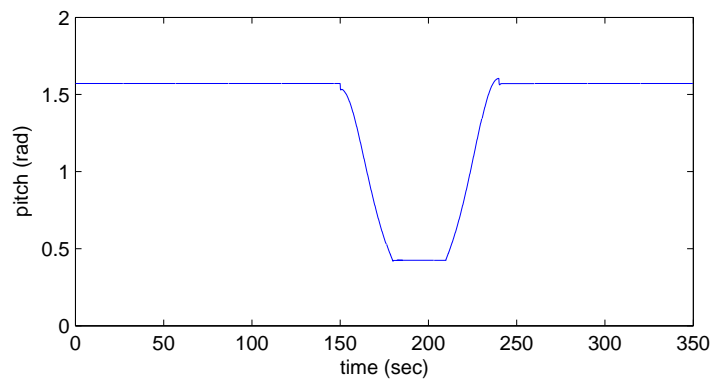

(a) Pitch angle

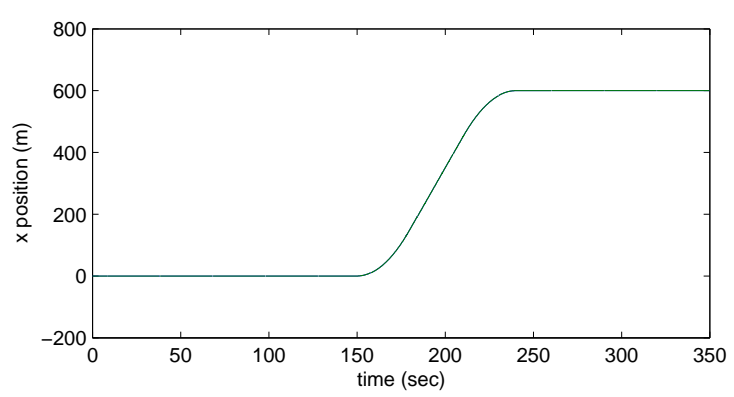

(c) $x$ position

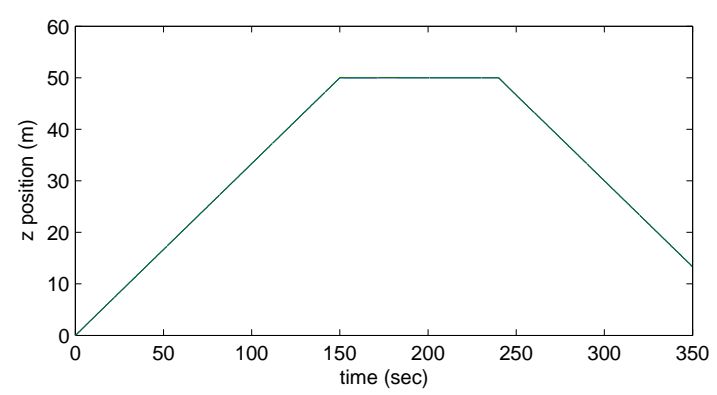

(e) $z$ position

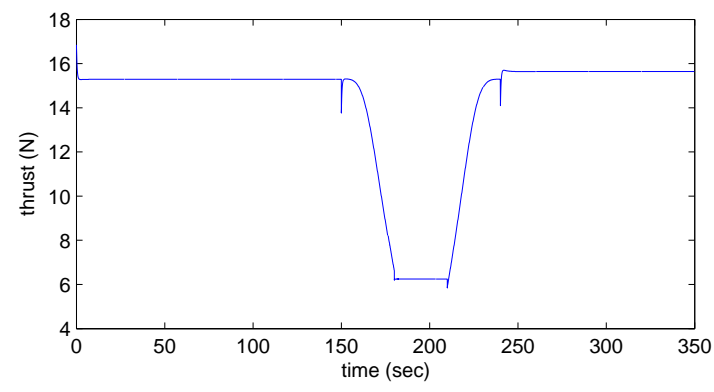

(b) Thrust

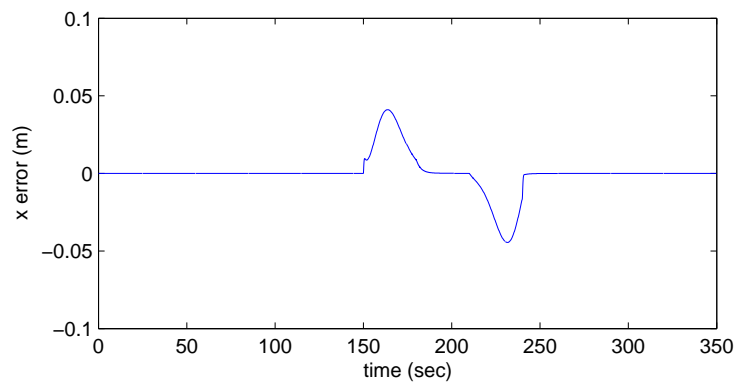

(d) $x$ position error

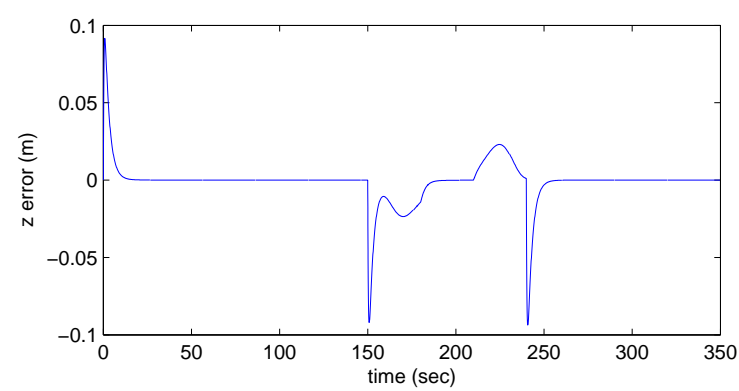

(f) $z$ position error

Figure 7. Simulation results of trajectory generation

\section{Conclusion}

In this paper we have outlined and explored some preliminary design concepts as well as given motivation for developing a tailsitter UAV. The paper has presented a simplified aerodynamic model for hover forces and torques, established a quaternion based attitude and position hover controller, put forward a method for following desired trajectories and proposed a hardware platform for physical implementation of these ideas. Simulation results have also been given for concept verification. The concepts discussed in this paper are preliminary in nature and will serve as a foundation for future work. Further research will focus on obtaining actual flight data for a tailsitter UAV.

\section{Acknowledgments}

This research was funded by AFRL/MN award no. FA8651-05-1-0006.

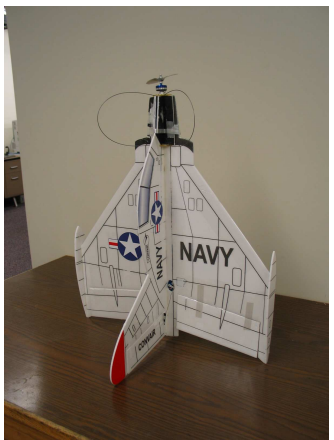

Figure 9. Model Pogo. 


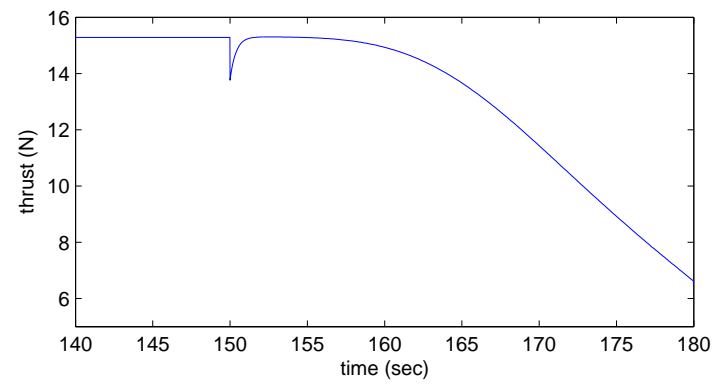

(a) Thrust - Vertical to level transition

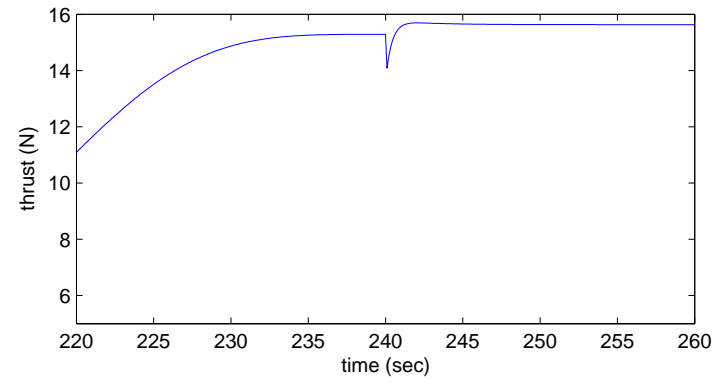

(b) Thrust - Level to vertical transition

Figure 8. Simulation results of trajectory generation - detail of thrust

\section{References}

${ }^{1} \mathrm{~K}$. Wernicke, "The single-propeller driven tailsitter is the simplest and most efficient configuration for the VTOL UAV's," in International Powered Lift Conference, 2000.

${ }^{2}$ A. Ailon, "Control of a vtol aircraft: Motion planning and trajectory tracking," in Mediterranean Conference on Control and Automation, 2005, pp. 1493-1498.

${ }^{3}$ P. Martin, S. Devasia, and B. Paden, "A different look at output tracking: control of a VTOL aircraft," in Conference on Decision and Control, 1994, pp. 2376-2381.

${ }^{4}$ R. Stone, "Control architecture for a tail-sitter unmanned air vehicle," in 5th Asian Control Conference, 2004, pp. 736-744.

5 __ "The T-wing tail-sitter unmanned air vehicle: from design concept to research flight vehicle," in Proceedings of the I MECH E Part G Journal of Aerospace Engineering, 2004, pp. 417-433.

${ }^{6}$ R. Stone and G. Clarke, "The T-wing: A VTOL UAV for defense and civilian applications," in UAV Australia Conference, 2001.

7 _ "Optimization of transition maneuvers for a tail-sitter unmanned air vehicle (UAV)," in Australian International Aerospace Congress, 2001.

${ }^{8}$ T. C. D J Taylor, M V Ol, "Skytote advanced cargo delivery system," in AIAA/ICAS International Air and Space Symposium and Exposition: The Next 100 Years, 2003.

${ }^{9}$ W. F. Phillips, Mechanics of Flight. New Jersey: Wiley, 2004

${ }^{10}$ H. W. B.Wei and A. Arapostathis, "Quaternion feedback regulator for spacecraft eigenaxis rotations," AIAA Journal of Guidence and Control, vol. 12, no. 3, 1989.

${ }^{11}$ A. G. K. S.M Joshi and J. T.-Y. Wen, "Robust attitude stabilization of spacecraft using nonlinear quaternion feedback," IEEE Transactions on Automatic Control, vol. 40, no. 10, 1995.

${ }^{12}$ R. Bach and R. Paielli, "Linearization of attitude-control error dynamics," IEEE Transactions on Automatic Control, vol. 38, no. 10, Oct. 1993

${ }^{13}$ W. Phillips, C. Hailey, and G. Gebert, "A review of attitude representations used for aircraft kinematics," Journal of Aircraft, vol. 38, no. 4, 2001.

${ }^{14}$ M. Shuster, "A survey of attitude representations," Journal of the Astronautical Sciences, vol. 41, no. 4, 1993.

${ }^{15}$ B. Stevens and F. Lewis, Aircraft Control and Simulation. Addison-Wesley Publishing Company John Wiley and Sons, Inc., 2003.

${ }^{16}$ R. E. Sheldahl and P. C. Klimas, "Aerodynamic characteristics of seven symmetrical airfoil sections through 180-degree angle of attack for use in aerodynamic analysis of vertical axis wind turbines," Sandia National Laboratories, Tech. Rep. SAND80-2114, 1980.

${ }^{17} \mathrm{http}: / /$ www.hobby-lobby.com/pogo.htm.

${ }^{18} \mathrm{http}: / /$ procerusuav.com/. 